\title{
Study on Biodegradation Characteristics of Aryl Organophosphorus Flame Retardants (aryl-OPFRs) by Sphingobium yanoikuyae YC-XJ2
}

\section{Xianjun Li}

graduate school of chinese academy of agricultural science

Junhuan Wang

graduate school of chinese acadeny of agricultural science

Thet Su Hlaing

graduate school of chinese academy of agricultural science

Yang Jia

graduate school of chinese academy of agricultural science

Yanchun Yan ( $\nabla$ yanyanchun2012@qq.com)

Graduate school of chinese academy of agricultural science,Beijing,100081,China https://orcid.org/0000-0002-9590-7646

\section{Research}

Keywords: Aryl organophosphorus flame retardants, Triphenyl phosphate, Biodegradation, Sphingobium yanoikuyae

Posted Date: December 10th, 2019

DOI: https://doi.org/10.21203/rs.2.18383/v1

License: (c) (1) This work is licensed under a Creative Commons Attribution 4.0 International License. Read Full License 


\section{Abstract}

The preprint has been retracted as all authors did not agree to posting.

\section{Preprint Retracted}

This preprint has been retracted as it was posted in error. 